\title{
Practice of Covid-19 Prevention Measure Inconsistence Definitions
}

\author{
Kaleab Tesfaye Tegegne ${ }^{1 *}$, Eleni Tesfaye Tegegne ${ }^{2}$ and Mekibib Kassa Tessema ${ }^{3}$ \\ ${ }^{1}$ Department of Public Health, Hawassa College of Health Science, Ethiopia \\ ${ }^{2}$ College of Medicine and Health Science, University of Gondar, Ethiopia \\ ${ }^{3}$ Leishmania Research and Treatment Center, University of Gondar, Ethiopia \\ *Corresponding author: Kaleab Tesfaye Tegegne MPH, Department of Public Health, Hawassa College of Health Science, Hawassa, \\ Ethiopia. \\ To Cite This Article: Kaleab Tesfaye Tegegne, Eleni Tesfaye Tegegne, Mekibib Kassa Tessema. Practice of Covid-19 Prevention Measure \\ Inconsistence Definitions. Am J Biomed Sci \& Res. 2021 - 13(2). AJBSR.MS.ID.001846. DOI: 10.34297/AJBSR.2021.13.001846.
}

Received: 眥 May 31, 2021; Published: 䤈 June 11, 2021

\section{Introduction}

According to WHO COVID-19 prevention measures include hand washing, wear mask, keeping distance, ventilated room, avoid crowding, and covering nose during coughing, avoid contact with animals and avoid consumption of raw meat [1,2]. Poor practices of hand hygiene, overcrowding and handshaking leads to increased spread of virus over short period [3]. A COVID-19 vaccine is a vaccine that provide acquired immunity against coronavirus 2 (SARS-CoV-2)) [4]. The WHO and the US CDC recommend individuals wear mask in public areas where there is an increased risk of transmission and where social distancing are difficult to maintain [5,6]. Crowded indoor spaces should be avoided [7]. The WHO recommends ventilation and air filtration in public spaces to help clear out infectious aerosols [8-10]. The WHO also recommends that individuals wash hands with soap and water for twenty seconds, after going to toilet or when hands are dirty, before eating and after blowing nose [11]. Individuals may apply social distancing methods by staying home, decrease travel, avoiding crowding, using no-contact meetings, and physically distancing from others [12]. The Harvard T.H. Chan School of Public Health recommends a healthy diet, physically activity, managing psychological stress, and getting enough sleep [13]. Different studies in various countries used different definitions to measure people's prevention practice of COVID-19.

\section{Inconsistence Definitions}

Study in Dire Dawa City, Eastern Ethiopia Used Six questions to assessed practice of COVID-19 prevention measure and those who scored less than mean were measured as having poor practice and those who scored a mean point above as having a good practice [14]. Study in Addis Ababa, Ethiopia used four items to assessed practice of COVID-19 prevention measure A score $75 \%$ and above as having a good practice and score $50 \%$ and below indicated a poor practice [15]. Study in Amhara region, Ethiopia: assessed A health workers practice of COVID-19 prevention measure, using a value of those scored $75 \%$ and above was grouped as "good practitioner" and who scored below 75\% was grouped as "poor practitioner" [16]. Study in Chinese residents: assessed A health workers practice of COVID-19 prevention measure by asking 2 things one for went to an overcrowded area and two wearing a mask when going out in recent days [17].

In another study: practice of COVID-19 prevention measure was assessed using Ten practice related questions with sixteen responses were asked. Those having scored $\geq$ mean by categorizing the clients as having good practice those who score less than the mean was classified as having poor practice [18]. As stated above different studies used different definitions to measure people's prevention practice of COVID-19 and this makes the analysis difficult as well as difficult to compare prevention practice of COVID-19 across countries.

\section{Conclusion}

We recommend researcher to develop a validated tool to measure prevention practice of COVID-19 and to study prevention practice of COVID-19 using health belief model or qualitative 
methods this is important to understand the real people's prevention practice of COVID-19.

\section{References}

1. (2020) World Health Organization. Coronavirus disease (COVID-19) advice for the public.

2. Brown A, Horton R (2020) A planetary health perspective on COVID-19: a call for papers. Lancet 395(10230): 1099.

3. (2020) National Comprehensive Covid19 Management Handbook FMOH pp.1-102.

4. Li YD, Chi WY, Su JH, Ferrall L, Hung CF, et al. (2020) Coronavirus vaccine development: from SARS and MERS to COVID-19. J Biomed Sci 27(1): 104.

5. Kate Kelland (2020) Wear masks in public says WHO, in update of COVID-19 advice.

6. (2019) Recommendation Regarding the Use of Cloth Face Coverings, Especially in Areas of Significant Community-Based Transmission. US Centers for Disease Control and Prevention (CDC).

7. (2020) Scientific Brief: SARS-CoV-2 Transmission. Centers for Disease Control and Prevention (CDC)

8. (2020) COVID-19 Employer Information for Office Buildings. National Center for Immunization and Respiratory Diseases (NCIRD). US Centers for Disease Control and Prevention (CDC)

9. (2020) WHO's Science in 5 on COVID-19 -Ventilation. World Health Organization.

10. Somsen GA, van Rijn C, Kooij S, Bem RA, Bonn D (2020) Small droplet aerosols in poorly ventilated spaces and SARS-CoV-2 transmission. Lancet Respir Med 8(7): 658-659.
11. (2020) Advice for the public on COVID-19 - World Health Organization. World Health Organization (WHO).

12. Nussbaumer SB, Mayr V, Dobrescu AI, Chapman A, Persad E, et al. (2020) Quarantine alone or in combination with other public health measures to control COVID-19: a rapid review. Cochrane Database Syst Rev 4(4): CD013574.

13. (2020) Food safety, nutrition, and wellness during COVID-19. The Nutrition Source. Harvard TH Chan School of Public Health.

14. Amsalu, Alemu G, Zinabie S, Nigus K, Alekaw S, et al. (2021) Practice of COVID-19 Prevention Measures and Associated Factors Among Residents of Dire Dawa City, Eastern Ethiopia: Community-Based Study. J Multidiscip Healthc 14: 219-228.

15. Defar A, Molla G, Abdella S, Tessema M, Ahmed M, et al. (2021) Knowledge, practiceand associated factors towards the prevention of COVID-19among high-risk groups: Across-sectional study in Addis Ababa, Ethiopia. PloS ONE 16(3): e0248420.

16. Asemahagn (2020) Factors determining the knowledge and prevention practice of healthcare workers towards COVID-19 in Amhara region, Ethiopia: a cross-sectional survey. Tropical Medicine and Health 48: 72.

17. Zhong BL, Luo W, Li HM, Qian Qian Zhang, Xiao Ge Liu, et al. (2020) Knowledge, attitudes, and practices towards COVID-19 among Chinese residents during the rapid rise period of the COVID-19 outbreak: a quick online cross-sectional survey. Int J Biol Sci 16(10): 1745-1752.

18. Ali Fakhari, Roya Dolatkhah, Keivan Fakhari Dehkharghani (2020) How the COVID-19 outbreak affected physician-patient relationship. J Community Med Health Solut 1: 023-025. 